\title{
FRAMEFIELDS AND NONDEGENERATE SINGULARITIES ${ }^{1}$
}

\author{
BY ULRICH KOSCHORKE
}

Communicated by Glen Bredon, October 1, $1974^{2}$

1. Introduction. In this note we outline an obstruction theory for vector bundle monomorphisms over compact manifolds. The basic idea is to proceed by induction over the rank of a bundle homomorphism, and to analyze arising singularities in order to decide whether the minimum rank can be increased. We carry this program out in a range of dimensions ("metastable") where the primary obstruction is the only one and where it gives complete existence and classification results.

Possible applications include planefields, foliations, immersions and some aspects of homotopy theory. Here we will deal only with framefields. Among other things the following interesting fact emerges from the discussion: while odd torsion invariants have no importance in many existence questions (Theorem 3), they play a significant role in the classification up to homotopy or up to certain bordism relations (see Theorem 4; however for concordance compare with the classification theorem of \$2).

The obstruction theory presented here was developed from earlier cruder (bordism) versions [2], [3]. Subsequently I learned that a stable variant of the existence theorem was previously proved by Salomonsen [5] by different methods.

2. An obstruction theory for bundle monomorphisms. We will denote the $r$ th bordism group of a space $X$ with coefficients in a virtual vector bundle $\phi$ by $\Omega_{r}(X ; \phi)$ (as in [5], except for the sign of $\phi$ ). Usually this group can be interpreted as a (stable) homotopy group of a suitable Thomspace.

Now let $\alpha, \beta$ be a real vector bundles of dimension $q$, resp. $m$, over a compact smooth $n$-manifold $M$, and let $u^{\prime}: \alpha|\partial M \subset \beta| \partial M$ be injective. If $2(m-q) \geqslant n-2$, we can extend $u^{\prime}$ to a homomorphism $u: \alpha \rightarrow \beta$ (over all of $M$ ) which is monomorphic outside of a nondegenerate singularity $S$ and such that at every point of $S$ the kernel of $u$ is 1-dimensional (compare

AMS (MOS) subject classifications (1970). Primary 57D25, 55G35, 57D90.

${ }_{1}^{1}$ Research partially supported by NSF Grant GP 38215.

${ }^{2}$ Originally received July $14,1974$.

Copyright $\odot 1975$, American Mathematical Society 
[2]). The following data can be extracted from the behavior of $u$ around $S$ : (i) a "kernel map" $g$ from $S$ into the projective space bundle $P(\alpha)$ associated with $\alpha$; and (ii) a homotopy class of stable isomorphisms $\bar{g}$ : TS $\oplus$ $g^{*}\left(\lambda \otimes \pi^{*}(\beta)\right) \rightarrow g^{*}(T(P(\alpha)))$, where $\pi: P(\alpha) \rightarrow M$ denotes the bundle projection, and $\lambda \subset \pi^{*}(\alpha)$ is the canonical line bundle over $P(\alpha)$. The bordism class of $(S, g, \bar{g})$ defines an invariant

$$
\omega\left(M, \alpha, \beta, u^{\prime}\right) \in \Omega_{n-m+q-1}\left(P(\alpha) ; \lambda \otimes \pi^{*}(\beta)-\lambda \otimes \pi^{*}(\alpha)-\pi^{*}(T M)\right) .
$$

It is not hard to obtain the classical (cohomology) primary obstruction for our extension problem from $\omega\left(M, \alpha, \beta, u^{\prime}\right)$.

Existence Theorem. Assume $2(m-q)>n$. Then $u^{\prime}$ can be extended to a monomorphism $u: \alpha \hookrightarrow \beta$ over all of $M$ iff $\omega\left(M, \alpha, \beta, u^{\prime}\right)=0$.

Classification Theorem. Let $2(m-q)>n+1$ (resp. $>n)$ and let a fixed monomorphism $u_{0}: \alpha \hookrightarrow \beta$ (extending $u^{\prime}$ ) be given. Then the homotopy (resp. concordance) classes of all such monomorphisms are in bijective correspondence with the elements of the group

$$
\Omega_{n-m+q}\left(P(\alpha) ; \lambda \otimes \pi^{*}(\beta)-\lambda \otimes \pi^{*}(\alpha)-\pi^{*}(T M)\right)
$$

(resp. of a quotient consisting entirely of 2-torsion).

Here concordance means homotopy through $\beta \oplus \mathbf{R}$.

EXAMPle 1 (STIEFEl MANIFOLdS AND STUNTED PROJECTIVE SPACES). Put $M=D^{n}, \alpha=\mathbf{R}^{q}$ and $\beta=\mathbf{R}^{m}$. Assigning to $\left[u^{\prime}\right] \in \pi_{n-1}\left(V_{m, q}\right)$ the invariant $\omega\left(D^{n}, \alpha, \beta, u^{\prime}\right)$, we obtain a "singularity isomorphism"

$$
\sigma: \pi_{n-1}\left(V_{m, q}\right) \rightarrow \Omega_{n-m+q-1}\left(p^{q-1} ;(m-q) \lambda\right)=\pi_{n-1}\left(P^{m-1} / P^{m-q-1}\right)
$$

for $2(m-q)>n$.

3. Invariants for framefields. Throughout this section let $M$ be a closed, connected smooth (oriented or nonorientable) $n$-manifold, and fix $0<q<n / 2$.

Consider the commutative diagram

$$
\pi_{n-1}\left(V_{n, q}\right) \underset{\Theta_{M}{ }_{\Omega_{q-1}}\left(P^{q-1} \times M ; \phi_{M}\right)}{\stackrel{\Theta}{\tau_{M}}} \Omega_{q-1}\left(P^{q-1} \times B(S) O(n) ; \phi\right)
$$


where $\phi=\lambda \otimes \gamma^{n}-q \cdot \lambda-\gamma^{n}$ and $\phi_{M}=\lambda \otimes T M-q \lambda-T M ; \Theta, \Theta_{M}$ and $\tau_{M}$ are obtained from $\sigma$ (see Example 1) and obvious inclusions or classifying maps.

Now define $\omega_{q}(M)=\omega\left(M, \mathbf{R}^{q}, T M\right) \in \Omega_{q-1}\left(P^{q-1} \times M ; \phi_{M}\right)$ and $\omega_{q}^{\prime}(M)=\tau_{M}\left(\omega_{q}(M)\right) \in \Omega_{q-1}\left(P^{q-1} \times B(S) O(n) ; \phi\right)$. If $u$ is a $q$-field with finite singularities on $M$, then $\operatorname{Index}(u) \in \pi_{n-1}\left(V_{n, q}\right)$ (in the sense of Thomas) gets mapped into $\omega_{q}(M)$ under $\Theta_{M}$.

THEOREM 1. $M$ admits a q-field without (resp. with finite) singularities iff $\omega_{q}(M)$ (resp. its class in the cokernel of $\Theta_{M}$ ) vanishes. $M$ is bordant (in Reinhart's refined bordism group $\mathfrak{M}_{0}^{n}$ or $\Omega_{0}^{n}$ ) to a manifold with a $q$ field iff $\omega_{q}^{\prime}(M)=0$.

THEOREM 2. Let $\theta: \pi_{n-1}\left(V_{n, q}\right) \rightarrow G$ be a homomorphism into a group $G$ such that for any q-field $u$ with finite singularities on a closed $n$-manifold $M, \theta$ (Index $(u)$ ) depends only on $M$ (and not on $u$ ). Then $\theta$ factors through $\Theta: \pi_{n-1}\left(V_{n, q}\right) \rightarrow$ image $(\Theta)$. Hence $\theta$ (Index $(u)$ ) depends only on the following invariants of $M$ : the Stiefel-Whitney numbers, the Euler number, and, in the oriented case, the Pontriagin numbers and (if $n \equiv 1$ (4)) the real Kervaire semicharacteristic.

REmarK. The kernel of $\Theta$ can be nontrivial (e.g. for $q \geqslant 4, n \equiv 3$ (8)), but it consists always only of 2 -torsion.

THEOREM 3. If $M$ has zero Euler number, then $2^{q-1} \cdot \omega_{q}(M)=0$. If in addition $u$ is a q-field with finite singularities on $M$, then $\operatorname{Index}(u)$ lies in the 2-torsion of $\pi_{n-1}\left(V_{n, q}\right)$.

For related results see work of Becker, Salomonsen [5], a.o.

Next let $v$ be a $(q-1)$-field without singularities on $M$. Define $\chi(M, v)$ e $\pi_{q-1}^{S}\left(M^{+}\right)=\Omega_{q-1}(M ;$ trivial $)$ to equal the obstruction $\omega(M, \mathbf{R}, \eta)$, where $\eta$ is a complement of the image of $v$ in $T M$. Using the obvious forgetful maps, define also $\chi^{\prime}(M, v) e \pi_{q-1}^{S}\left(B(S) O(n)^{+}\right)$and $\chi^{\prime \prime}(M, v) e \pi_{q-1}^{S}$. The invariant $\chi(M, v)$ (resp. $\chi^{\prime}(M, v)$ ) vanishes iff $v$ has a complementary vector field (resp. has one after a suitable bordism).

TheOREM 4. Assume that $M$ admits $a(q-1)$-field. Then the invariant $\chi^{\prime \prime}(M, \cdot)$ detects at least as many different (bordism) classes of $(q-1)$ fields on $M$ as there are elements in the kernel of $i_{*}: \pi_{n-1}\left(S^{n-q}\right) \rightarrow$ $\pi_{n-1}\left(V_{n, q}\right)$. Note that for $n \neq \equiv q$ (2) the odd torsion of $\pi_{n-1}\left(S^{n-q}\right) \cong \pi_{q-1}^{S}$ lies in this kernel. 
REMARK. Ed Miller has also studied $\chi(M, v)$ and $\chi^{\prime \prime}(M, v)$.

\section{REFERENCES}

1. M. Atiyah and J. Dupont, Vector fields with finite singularities, Acta Math. 128 (1972), 1-40.

2. U. Koschorke, Singularities and bordism of q-plane fields and of foliations, Bull. Amer. Math. Soc. 80 (1974), 760-765.

3. - Bordism of immersions and k-mersions; Rational bordism of frame fields, immersions and k-mersions, Notices Amer. Math. Soc. 21 (1974), A-17, A-405. Abstracts \#74T-G12; 713-G5.

4. - Existence and classification of frame fields, Notices Amer. Math. Soc. 21 (1974), A-508. Abstract \#74T-G102 and A-563.

5. H. A. Solomonsen, Bordism and geometric dimension, Math. Scand. 32 (1973), 87-111.

DEPARTMENT OF MATHEMATICS, QUEENS COLLEGE, CUNY, FLUSHING, NEW YORK 11367 